\title{
EQUIVALÊNCIA ENTRE DADOS DE TRAÇÃO A QUENTE E FLUÊNCIA PARA O COBRE COMERCIAL PURO. PARTE 2: ANÁLISE POR DIFERENTES METODOLOGIAS DE PARAMETRIZAÇÃO
}

Levi de Oliveira Bueno ' Cayo Roberto Ferreira Dias ${ }^{2}$

\section{Resumo}

Investiga-se a possibilidade de parametrização de dados de tração a quente juntamente com dados de fluência, no cobre comercial puro, quando se faz a sua conversão segundo um critério de equivalência proposto recentemente para análise de resultados dessas duas modalidades de teste. Os dados são analisados por quatro diferentes métodos comumente citados na literatura tradicional de parametrização de dados de fluência. Observa-se boa compatibilidade dos resultados de tração a quente com os de fluência, quando analisados pelos métodos de Orr-Sherby-Dorn, MansonSuccop e Manson-Haferd, com possibilidade de se utilizar ambos os tipos de resultados em conjunto em procedimentos de extrapolação. A melhor compatibilidade é obtida com o procedimento de Manson-Haferd, que deixa os dados dos dois tipos de teste praticamente indistintos, produzindo uma curva paramétrica de alta qualidade. Por outro lado, o método mais conhecido de Larson-Miller não é eficaz na racionalização conjunta dos dados dos dois grupos de teste, utilizando-se uma única constante $C$ de parametrização.

Palavras-chave: Tração a quente; Fluência; Correlação; Parametrização.

\section{EQUIVALENCE BETWEEN HOT TENSILE AND CREEP TESTS FOR COMMERCIALLY PURE COPPER. PART 2: ANALYSIS BY DIFFERENT PARAMETERIZATION METHODOLOGIES}

\begin{abstract}
The possibility of parameterization of hot tensile data together with creep data is investigated, for commercially pure copper, according to a recent criterion that pro-poses an equivalence for both kinds of tests. Four different parameterization methods are considered, which are commonly mentioned in the traditional literature for data extrapolation. The hot tensile data shows good compatibility with creep data when the analysis is performed by the Orr-Sherby-Dorn, Manson-Succop and Manson-Haferd procedures. The Manson-Haferd method rationalizes the results so well that make the distinction between both kinds of data almost impossible, producing a remarkably good quality reference curve. On the other hand, the more popular Larson-Miller method is not able to generate a consistent reference curve by the use of a single $C$ constant for both data.
\end{abstract}

Key words: Hot tensile; Creep; Correlation; Parameterization.

\footnotetext{
' PhD em Engenharia de Materiais, Professor Adjunto do DEMa / UFSCar, Rodovia Washington Luís, km 235 - SP-310, Cep 13565-905, São Carlos, SP, Brasil. E-mail: levi@ufscar.br

${ }^{2}$ Licenciado em Física, Estudante de mestrado do PPG / CEM, UFSCar, Rua Paschoal Contieiro, 190 - Vila São Jorge, Cep 137 I0-000,

Tambaú, SP, Brasil. E-mail: cayodias@hotmail.com
} 


\section{INTRODUÇÃO}

\section{I.I Apresentação}

Este artigo representa uma continuação do trabalho de análise de resultados apresentados na Parte I desta publicação, investigando agora a possibilidade de correlação entre os dados de tração a quente com os de ruptura por fluência no cobre policristalino comercialmente puro, utilizando o critério de equivalência entre esses dois tipos de teste, proposto por Bueno. (1)

No presente trabalho, explora-se a possibilidade de parametrização conjunta dos dados, segundo diferentes procedimentos de análise, dentre as técnicas tradicionais mais importantes mencionadas na literatura.

A maneira de se aplicar o critério na conversão de dados de tração a quente para fluência está apresentada com mais detalhes na Parte I deste trabalho.

\section{I.2 Objetivo}

O objetivo é, além de explorar a possibilidade de parametrização conjunta dos dados, verificar se é possível estabelecer uma correlação entre os resultados desses dois tipos de ensaio para o cobre, visando extrapolação dos resultados por diferentes metodologias. Essa correlação foi verificada com sucesso em diferentes tipos de aço, como reportada em trabalhos anteriores. ${ }^{(2-4)}$

Dentre as várias possibilidades citadas na literatura, são escolhidos apenas quatro métodos, considerados em geral como os mais importantes: Larson-Miller, Orr-Sherby-Dorn, Manson-Succop e Manson-Haferd.

\section{I.3 Revisão da Literatura}

\section{I.3.I Métodos de parametrização}

Existe uma série de métodos de parametrização que utilizam equações relacionando a temperatura e o tempo de ruptura para análise de dados de fluência. A definição dos parâmetros baseia-se na linearidade e padrão de disposição dos pontos de isotensão em gráficos de LOG (Tempo de Ruptura) versus Temperatura ou LOG (Tempo de Ruptura) versus o Inverso da Temperatura. Os diferentes métodos surgem pelas hipóteses de paralelismo ou convergência dessas linhas de isotensão, nesses gráficos.

De acordo com Viswanathan, ${ }^{(5)}$ os métodos de parametrização mais utilizados e considerados neste trabalho estão esquematizados na Figura I.

Cada método de parametrização possui uma expressão correspondente a seu parâmetro:

a) Método de Larson-Miller $P=T\left(C+\right.$ LOG $\left.t_{r}\right)$

b) Método de Orr-Sherby-Dorn $P=$ LOG $t_{r}-A / T$

c) Método de Manson-Haferd $P=\left(\right.$ LOG $t_{r}-$ LOGt*)/(T - T*)

d) Método de Manson-Succop P $=$ LOG $t_{r}-$ B.T

onde $t_{r}=$ tempo ruptura $(h), T=$ temperatura $(K), A$, $\mathrm{B}, \mathrm{C}, \mathrm{LOGt}^{*}$ e T* são as constantes de cada método. Os métodos de Larson-Miller e Manson-Haferd consideram convergência, enquanto que os métodos de Orr-Sherby-Dorn e Manson-Succop consideram paralelismo das linhas de isotensão, respectivamente nos espaços LOG $t_{r} \times I / T$ e LOG $t_{r} \times T$, como mostram os diagramas da Figura $I$.

\section{MATERIAIS E MÉTODOS}

O material utilizado neste trabalho é o mesmo apresentado no artigo da Parte I desta publicação, ou seja, trata-se de cobre policristalino, comercialmente puro, recebido na forma trefilada, com estrutura de grãos alongados por encruamento. $\mathrm{O}$ material foi recozido a $600^{\circ} \mathrm{C}$ por uma hora, com resfriamento rápido ao ar, sofrendo recristalização e crescimento de grão, exibindo finalmente estrutura granular equiaxial, com tamanho médio de grão $\mathrm{D}=80 \mu \mathrm{m}$. A resistência à tração também foi testada à temperatura ambiente antes e depois deste processo. Finalmente, o material foi considerado adequado para ser submetido aos ensaios de tração a quente e de fluência.

Os corpos de prova utilizados nos ensaios de tração e de fluência possuíam as mesmas medidas, sendo de formato cilíndrico com rosca nas extremidades tipo $M 8 \times 1,25$, e dimensões nominais: $d_{0}=4 \mathrm{~mm} \mathrm{e}_{0}=16 \mathrm{~mm}$, na parte útil.

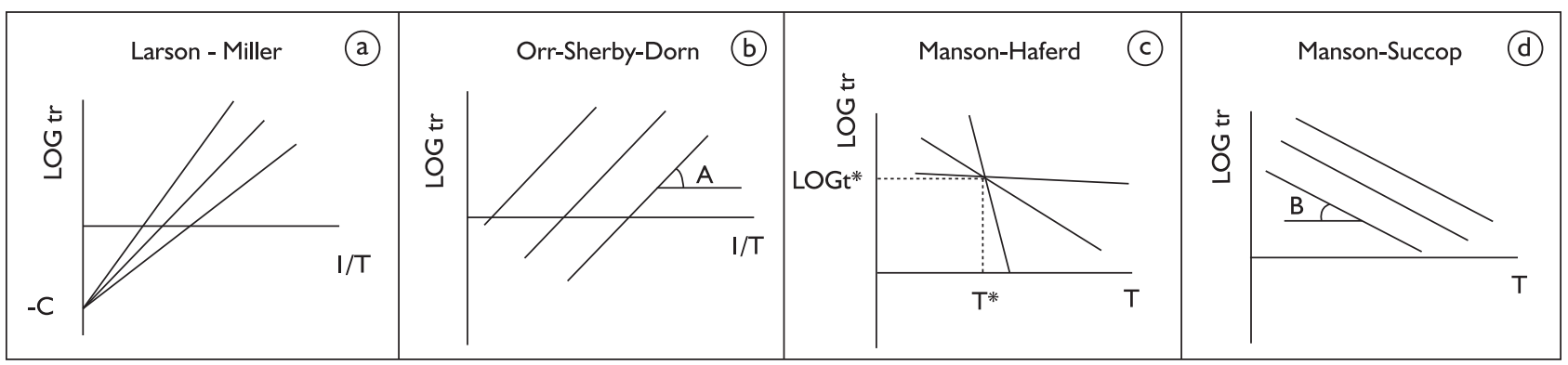

Figura I. Métodos de parametrização: a) Larson-Miller; b) Orr-Sherby-Dorn; c) Manson-Haferd; e d) Manson-Succop. ${ }^{(5)}$ 


\section{I Ensaios de Tração}

Esses ensaios foram realizados nas temperaturas de $300^{\circ} \mathrm{C}, 350^{\circ} \mathrm{C}, 400^{\circ} \mathrm{C}, 450^{\circ} \mathrm{C}$ e $500^{\circ} \mathrm{C}$, utilizando as velocidades de tração: $0,01 \mathrm{~mm} / \mathrm{min}, 0,8 \mathrm{~mm} / \mathrm{min}$, $20 \mathrm{~mm} / \mathrm{min}$, que correspondem, respectivamente, às taxas de deformação nominais médias $1,0 \times 10^{-5} \mathrm{~s}^{-1}, 8,3 \times 10^{-4} \mathrm{~s}^{-1} \mathrm{e}$ $0,0208 \mathrm{~s}^{-1}$. O equipamento utilizado foi uma máquina de marca TIME modelo WDW-I00E operando em conjunto com um forno elétrico tubular com resistências de Kanthal. Tanto para o controle da temperatura do forno, como para a medida da temperatura do corpo de prova foram utilizados termopares tipo Chromel-Alumel, sendo o primeiro colocado junto às resistências do aparelho, e o segundo na superfície da parte útil do corpo de prova.

\subsection{Ensaios de Fluência}

Os ensaios de fluência foram realizados na modalidade carga constante, variando de 7,5 $\mathrm{MPa}$ a $60 \mathrm{MPa}$, abrangendo 10 diferentes níveis de temperatura: $250^{\circ} \mathrm{C}$, $275^{\circ} \mathrm{C}, 300^{\circ} \mathrm{C}, 325^{\circ} \mathrm{C}, 350^{\circ} \mathrm{C}, 400^{\circ} \mathrm{C}, 450^{\circ} \mathrm{C}, 475^{\circ} \mathrm{C}$, $500^{\circ} \mathrm{C}$ e $525^{\circ} \mathrm{C}$, totalizando 21 ensaios. Empregou-se um conjunto de cerca de 10 máquinas de ensaio marca STM, modelo MF-1000. O sistema de extensometria nos corpos de prova utilizou sensores tipo LVDT associados a uma armazenadora automática de dados tipo Data Logger, marca Fluke. As medidas da variação do alongamento em função do tempo foram armazenadas em intervalos de tempo que variaram de aproximadamente 5 minutos a 30 minutos, sendo que este período dependia das previsões de duração de cada ensaio.

\section{RESULTADOS}

$\mathrm{Na}$ tentativa de relacionar os dados de tração a quente com os dados de fluência, foi construído o gráfico do LOG (Tensão) versus LOG (Tempo de Ruptura), também conhecido como gráfico da queda de resistência com o tempo, apresentado na Figura 2.

As Figuras 3 e 4 mostram o padrão de disposição das seis linhas de iso-tensão nos planos $\operatorname{LOG}\left(\mathrm{t}_{r}\right) \times 1 / T$ e $\operatorname{LOG}\left(\mathrm{t}_{\mathrm{r}}\right) \times \mathrm{T}$, respectivamente. Esses gráficos contêm apenas os dados de fluência, já que se torna impossível obter linhas de iso-tensão com os dados de tração a quente.

A maneira correta de se calcular as constantes envolvidas em cada metodologia é por um procedimento de mínimos quadrados, como o recomendado por Manson e Mendelsohn. ${ }^{(6)}$ Como o trabalho ainda está em desenvolvimento, são tomados apenas os valores correspondentes à média dos pontos de interseção e de inclinação das retas de isotensão, que fornecem valores aproximados dos reais. Assim que todos os testes forem finalizados 0

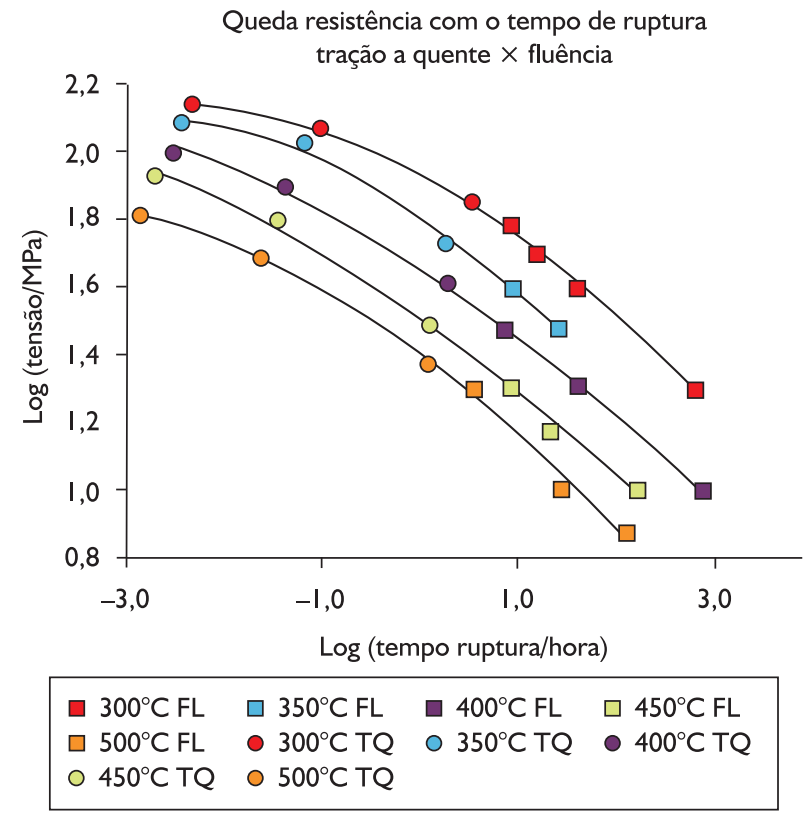

Figura 2. Gráfico da queda de resistência com o tempo para os dados de tração a quente e de fluência. Obs: $\mathrm{TQ}=$ tração a quente; $\mathrm{FL}=$ fluência.

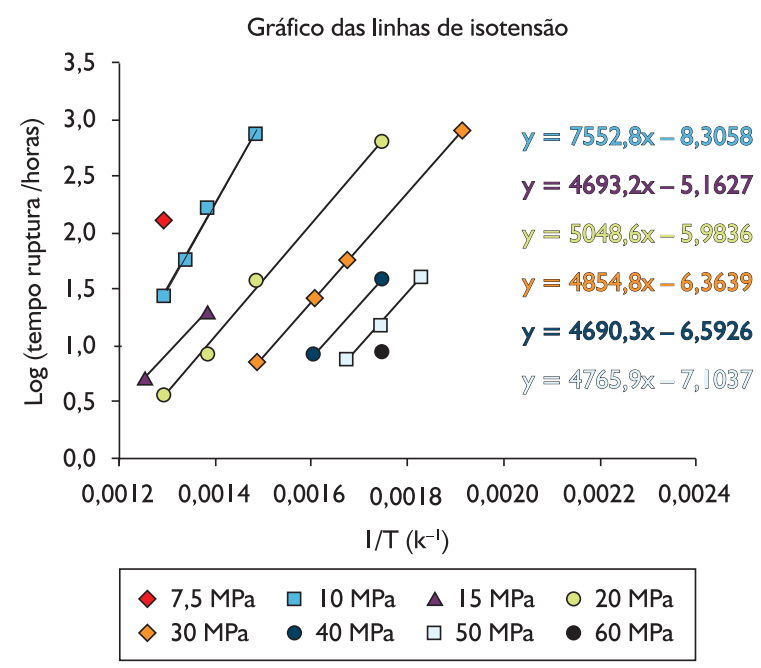

Figura 3. Linhas de isotensão no espaço $\operatorname{LOG}\left(\mathrm{t}_{\mathrm{r}}\right) \times \mathrm{I} / \mathrm{T}$, utilizado para a determinação das constantes dos métodos de Larson-Miller e Orr-Sherby-Dorn.

procedimento( ${ }^{(6)}$ será aplicado na íntegra. No caso da verificação do método de Manson-Haferd, nota-se que o foco das linhas pode estar bem próximo do eixo $Y\left[\operatorname{LOG}\left(\mathrm{t}_{\mathrm{r}}\right)\right]$. Portanto, nesse caso, toma-se o valor $\mathrm{T}^{*}=0$. 


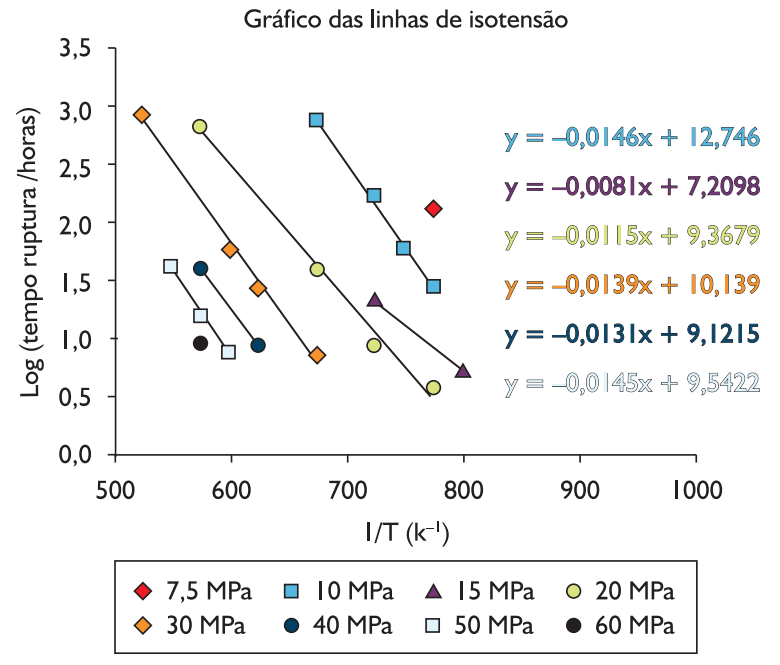

Figura 4. Linhas de isotensão no espaço LOG(tr) $x$ T, utilizado para a determinação das constantes dos métodos de Manson-Succop e Manson-Haferd.

Com a determinação das várias constantes, foram obtidas as curvas de referência, segundo cada método. A Figura 5 corresponde à parametrização dos dados apresentados na Figura I, segundo o método de Larson-Miller. Essa figura corresponde à utilização da constante $C=6,585$, que expressa o ponto focal médio do conjunto de linhas de isotensão no eixo $Y$.

Observa-se que os dados de fluência ficam muito bem parametrizados. No entanto, os dados de tração a quente continuam dispersos, alinhados segundo as diferentes temperaturas utilizadas nos testes. $O$ coeficiente de correlação $R^{2}=0,9668$ também não é satisfatório. Dessa forma, a metodologia Larson-Miller não é eficaz em correlacionar os dois grupos de dados em uma única curva de referência.

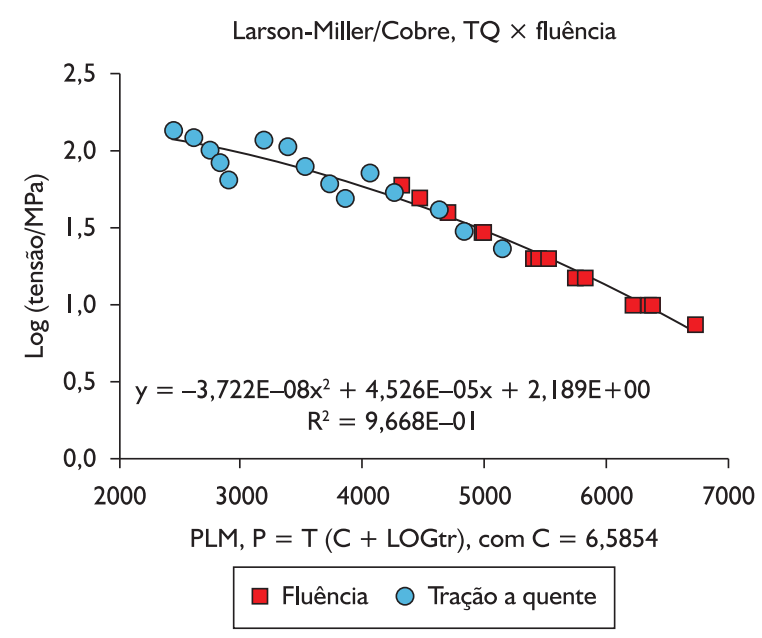

Figura 5. Curva paramétrica de Larson-Miller para $C=6,585$.
A Figura 6 apresenta a parametrização segundo o método de Orr-Sherby-Dorn. A constante utilizada foi $A=5.267,6$, que corresponde ao paralelismo médio das linhas de isotensão na Figura 3. A racionalização dos dois conjuntos de dados neste caso é bem melhor que no caso anterior, exibindo um valor de $\mathrm{R}^{2}=0,9859$.

A Figura 7 apresenta $\circ$ resultado de parametrização com o método de Manson-Succop. A constante desse método foi tomada como $B=-0,0126$, que corresponde também ao paralelismo das linhas de isotensão na Figura 4. A qualidade de ajuste para ambos os conjuntos de dados $\left(R^{2}=0,9885\right)$ também é muito boa, semelhante à obtida com o método de Orr-Sherby-Dorn.

$\mathrm{Na}$ última parametrização, foi utilizado o método de Manson-Haferd cujo resultado está representado na Figura 8. Como mencionado anteriormente, o valor de $\mathrm{T}^{*}$ é considerado como zero. $\mathrm{O}$ cálculo da intersecção

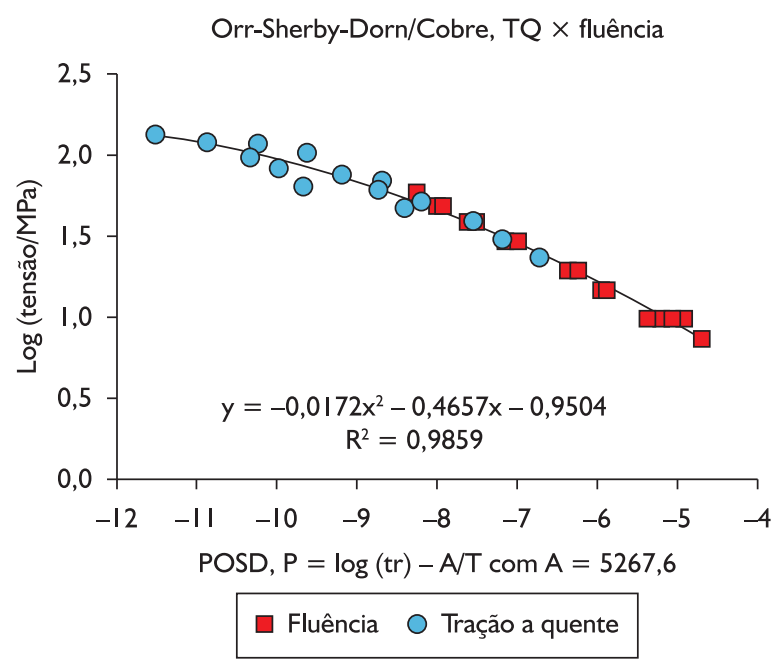

Figura 6. Curva paramétrica de Orr-Sherby-Dorn, com A = 5267,6.

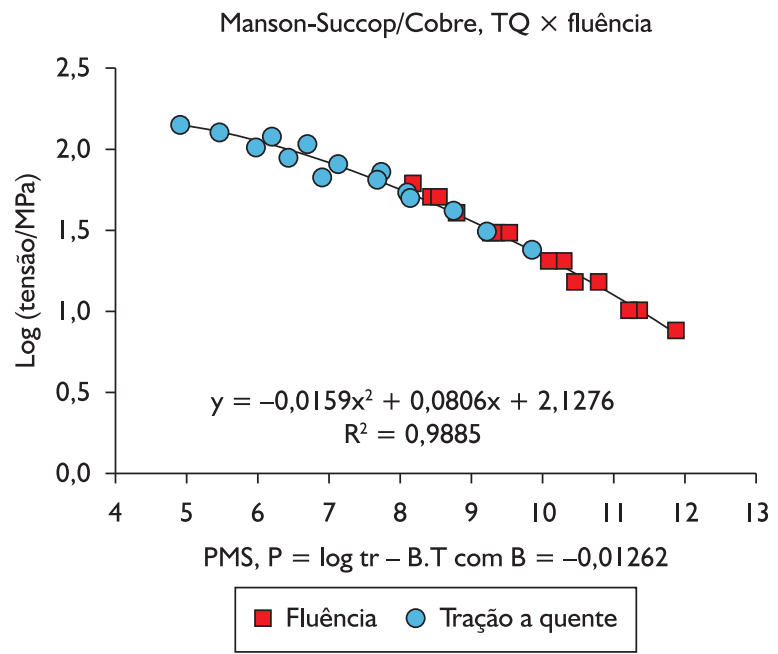

Figura 7. Curva paramétrica de Manson-Succop, com $B=-0,0126$. 


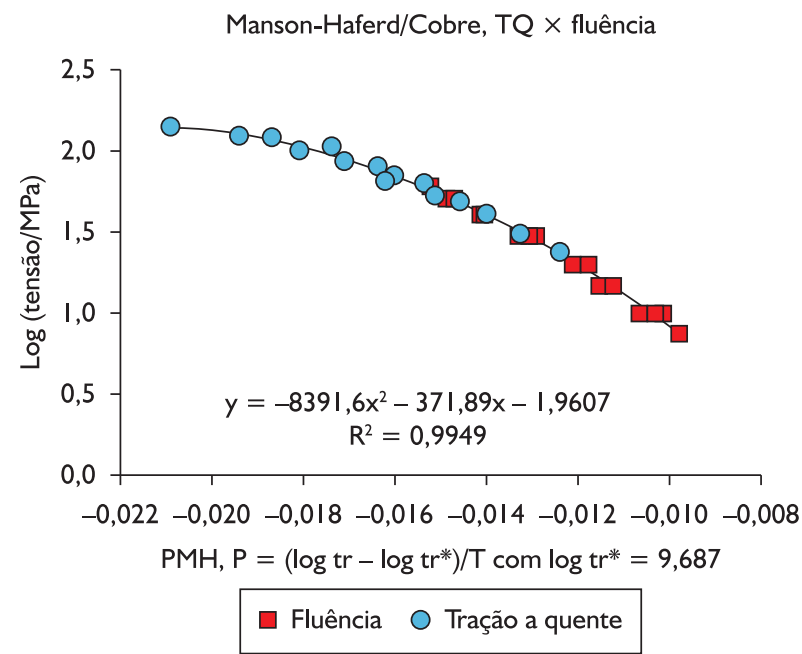

Figura 8. Curva paramétrica de Manson-Haferd, com $T^{*}=0$ e LOGt $_{r}^{*}=9,687$.

média com o eixo Y apresentou um valor LOGt ${ }_{r} *$ 9,687. Observa-se que a capacidade de racionalização de ambos os conjuntos de dados por este método é excelente, exibindo um grau de ajuste notável: $\mathrm{R}^{2}=0,9949$.

\section{DISCUSSÃO}

O gráfico da queda de resistência com o tempo mostrado na Figura 2 indica ótima concordância entre os dados de tração a quente e os dados de fluência em todos os níveis de temperatura. Este é um resultado muito importante, pois indica que os dados de tração a quente são totalmente compatíveis com os de ruptura por fluência, para o cobre policristalino comercialmente puro.

Na Parte I deste trabalho, comprova-se que esta compatibilidade ocorre para os dados obtidos basicamente no estágio secundário de fluência, que envolvem os valores de Taxa Mínima de Deformação. Com o presente trabalho, a compatibilidade notada entre os dados de tração a quente e os dados de Tempo de Ruptura em Fluência indica que a correspondência entre os dois tipos de teste se dá também durante o estágio terciário de fluência.
Com exceção do método de Larson-Miller, todos os outros métodos de parametrização, considerados para análise neste trabalho, mostram boa compatibilidade entre os dois grupos de testes, indicando consistência para seu futuro uso em técnicas de extrapolação de dados. Os métodos de Orr-sherby-Dorn e Manson-Succop mostram-se bastante eficazes, produzindo apenas uma certa dispersão nos dados de tração a quente, acompanhada por ótima racionalização dos dados de fluência. $\mathrm{O}$ método de Manson-Haferd apresenta os melhores resultados entre os quatro investigados. Tanto os grupos de dados de tração a quente como o de fluência apresentam um grau mínimo de dispersão, sendo excelente o grau de compatibilidade entre os dois tipos de dados.

\section{CONCLUSÃO}

O presente trabalho cumpre a contento o objetivo traçado inicialmente em tentar encontrar uma correlação satisfatória entre os dados de tração a quente e os dados de fluência, aplicando-se o critério(l) de equivalência proposto para análise conjunta de ambos os tipos de dados, com o cobre policristalino. Trabalhos anteriores já mostram a eficiência desse critério na conversão de dados para um aço ferrítico e um inoxidável austenítico. ${ }^{(2-4)}$ Este trabalho aponta para a possibilidade de se utilizar com sucesso o critério a fim de se estabelecer a correlação entre tração a quente e fluência também para os materiais não-ferrosos.

\section{Agradecimentos}

Os autores agradecem à empresa STM - Sistemas de Teste em Materiais Ltda., pelo apoio financeiro oferecido na obtenção do material, sua usinagem e realização dos testes de tração a quente e de fluência.

Cayo Roberto Ferreira Dias agradece ao Conselho Nacional de Desenvolvimento Científico e Tecnológico CNPq pela concessão da bolsa de Mestrado e aos técnicos Gustavo Pelissari, William de O. Paiva e Heber P. Cardoso, pelo apoio durante a execução dos ensaios mecânicos.

\section{REFERÊNCIAS}

I BUENO, L. O. Creep behaviour of $2.25 \mathrm{Cr}$-IMo steel: an equivalence between hot tensile and creep testing data. In: ECCC CREEP CONFERENCE, 2005, London. Creep \& fracture in high temperature components: design and life assessment issues. Lancaster: DEStech Publications, Inc., 2005. p. 969-80.

2 REIS SOBRINHO, J. F. Correlação entre os comportamentos de tração a quente e fluência do aço 2,25Cr-I Mo na faixa de temperatura de $500^{\circ} \mathrm{C}$ a $700^{\circ} \mathrm{C}$. 2004. 212 f. Tese (Doutorado em Ciência e Engenharia de Materiais) - Ciência e Engenharia de Materiais da Universidade Federal de São Carlos, São Carlos, 2004. 
3 SANTOS, N.B. Avaliação de um critério de equivalência entre dados de tração a quente e fluência em aços. 2007, $125 \mathrm{f}$. Dissertação (Mestrado em Ciência e Engenharia de Materiais) - Ciência e Engenharia de Materiais da Universidade Federal de São Carlos, São Carlos, 2007.

4 MORETO, J. A. Avaliação do desempenho de aços inoxidáveis ferríticos com relação a propriedades mecânicas e oxidação em altas temperaturas. 2008, 96 f. Dissertação (Mestrado em Engenharia e Ciência de Materiais) - Curso de Engenharia da Universidade Federal do Paraná, Curitiba, 2008.

5 VISWANATHAM. R. Damage mechanisms and life assessment of high temperature components. Ohio: ASM International, 1993.

6 MANSON, S. S.; MENDELSOHN, A. Optimization of parametric constants for creep-rupture data by means of least-squares. NASA Memorandum, n.3-10-59E, p. I-34, 1959.

Recebido em: 01/10/2010

Aceito em: 3l/03/20I I 